\title{
A Novel Approach for Detection of Optic Disc and Lesion Location for Screening Diabetic Retinopathy
}

\author{
Aakanksha Mahajan, Amity University, Noida, India \\ (iD) https://orcid.org/0000-0003-2775-7751 \\ Vasudha Vashisht, Amity University, Noida, India \\ Rohit Bansal, Rajiv Gandhi Institute of Petroleum Technology, Amethi, India
}

\begin{abstract}
Diabetic retinopathy is not typically perceivable in diabetic patients at the initial stage. Their first signs, like micro-aneurysms, often go unnoticed in preliminary testing by specialists. Additionally, its presence is difficult to detect as there are other pathologies that may also lead to induce similar signs and symptoms. Until the detection of the presence of exudates, a specialist cannot simply deduce the presence of diabetic retinopathy. This paper presents a method to assist in the identification and differentiation of exudates on colour retinal images based on a variety of k-nearest neighbour filters. The proposed method proved to be a rational approach to detect bright lesions with sufficient certainty, yielding a possible injury with a specificity of $99 \%$.
\end{abstract}

\section{KEYWORDS}

Circular Hough Transform (CHT), Diabetic Retinopathy (DR), Exudates, K-Nearest Neighbour (KNN), Retinal

\section{INTRODUCTION}

Retinography, one of the most used non-invasive medical techniques in the analysis of the human visual system, refers to the clinical procedures used by highly qualified specialists to capture images that are further trained for the analysis, screening and diagnosis of specific visual diseases. The goal of a diabetes screening program is to detect and diagnose diseases before they cause vision loss. The mass screening program utilizes computer fundus images of the retina with or without mydriasis (dilated pupil) to diagnose eye diseases. Automatic retinal analysis can improve the performance of a detection program over manual imaging.

Diabetes Retinopathy (DR) is a retinal disease caused by the long standing diabetes mellitus (DM) (Niemeijer, et al., 2009; Junior, et al., 2013; Rahim, et al., 2016;). DR is the leading cause of blindness in people between the ages of 20 and 60 . The likelihood of developing DR is $78 \%$ if the patient is over 30 years of age and has had diabetes for more than 15 years (Junior, et al., 2013; Marin et al., 2015). As a result of DR, the surface of the retina is severely damaged. Cotton wools, microaneurysms, exudates and haemorrhages are the primary lesions indicating the presence of DR. Microaneurysms (MAs) are lesions that appear at an early stage of the disease, and the number of MAs increases with the progression of the disease (Adal, et al., 2014; Rahim, et al., 2014). Haemorrhages are likely an injury that occurs after MAs. When the vascular tree of retina leaks blood, it forms haemorrhages 
(Jitpakdee, et al., 2012; Inbarathi \& Karthikeyan, 2014). Thus, the diagnosis of haemorrhages and MAs is an essential part of the automatic diagnosis of retinal diseases at an early stage (Fleming, et al., 2006; Bae, et al., 2011; Rahim et al., 2016). At a later stage, a yellowish-white plasma begins to flow from the blood vessels, which is called hard exudates (Walter et al., 2002). Along with the exudates, a cotton wool appeared - a silver stain caused by the leakage of fat from the vessels. Haemorrhages, MAs, and exudates are features of non-proliferative diabetic retinopathy (NDPR) which is an early stage of DR. An advanced stage of DR, referred to as proliferative diabetic retinopathy (PDR) (Shah \& Han, 2004; Danis \& Davis, 2008), can have the main blood vessels of the retina entirely blocked. To supply the oxygen, new blood vessels develop, called abnormal blood vessels (DRSRG, 1981; Welikala, et al., 2014). The patient's vision has a significant impact in PDR. The main problem with DR is its asymptomatic nature as it does not affect vision until it reaches a higher level. DR can affect one or both eyes at the same time.

Fundus color images of the retina include various structures such as blood vessels, optic disc, fovea and red lesions such as haemorrhages and MAs. Haemorrhages and MAs comes under dark lesions, while cotton wools and exudates are deliberated as bright lesions. To diagnose fundus imaging lesions, the retinal morphological structure must be removed. Mathematical morphology is utilized to find the location of fovea, vascular trees, and optic disc segmentation (Dougherty \& Lotufo, 2003).

In this paper, a method to assist in the identification and differentiation of exudates on colour retinal images based on a variety of k-NN filters is presented. First, we offer a state-of-the-art review on DR detection. Then, the proposed novel method is presented; following this, details on the evaluation performed for the comparable techniques are highlighted. Finally, we close the discussion with key insights on practical implications, limitations and other concluding remarks.

\section{STATE-OF-THE-ART REVIEW}

The automatic detection of eye diseases based on the formation of the fundus is now a vital topic for scientists. The automatic detection of glaucoma and DR have captured the attention of key researchers working in the fields of medical imaging. Various systems and algorithms have soon appeared for DR analysis over the past two decades. In the past, several methods have been proposed for processing retinal images. For example, Rasta, et al. (2015) and Yadav, et al. (2016) have compared between contrast enhancements and illumination correction techniques.

More specifically, we review herein the literature on various algorithms for automatic DR detection from fundus images. Welfer, et al. (2010) proposed a method for rescuing the optic nerve from eye color formation in mathematical morphology. In their article, the analysis of an optic disc is divided into two parts; the first partition finds points on the disk, and the second part finds the boundaries of the disk. They used the main pixel of the blood vessels close to the center of the disk as an "inner label" and a circle of different diameters centered on the disk as the "outer label".

Parvati, et al. (2008) presented a watershed transform based medical image segmentation. Initially, a morphological gradient is applied to the grayscale image to show the roughness of the edges of each pixel, which in turn generate internal and external signals with regional peaks. This tag is placed on the original image and then applied to the watershed transformation for segmentation. A common issue in watershed transformation is excessive segmentation, which arises from quantization and noise errors.

Rahim, et al. (2016) recommended automatic diagnosis of the microscopic pathogenesis of DR. They explore various pre-processing, feature extraction, and classification approaches. The first phase uses discrete wavelet transform (DWT), histogram equalization, filtering and morphological operations for pre-processing. The feature extraction of DR contains pixel area, mean and standard deviation. The second phase uses segmentation of vessel, color correction and morphological operations to achieve the pre-processing. They used radial basis function, support vector machine (SVM), k-nearest neighbour (kNN) and decision tree (DT) classifier. The third phase consists of only two steps: pre-processing and detection of MA. Pre-processing is achieved by histogram equalization 
and RGB to grey conversion. Circular Hough Transform (CHT) has been proposed to detect MA due to its circular shape. The fourth phase also has two steps. The first stage uses a combination of preprocessing methods; RGB to grey conversion and contrast limited adaptive histogram equalization (CLAHE) while the fuzzy based histogram equalization and grey scale are utilized in the second stage. Detection of MA is done by using CHT.

Sahlsten, et al. (2019) developed a deep learning system to more accurately define DR than existing studies. The analysis was carried out on a small part of the image with a higher resolution. Their results highlight the ability of deep learning models to diagnose diseases at desired performance levels while accommodating cost constraints. Krause, et al. (2018) applied a solution for quantifying errors in DR, which focused on the classification of diseases via a deep learning algorithm. Kappa values were measured and model performance compared based on sensitivity, accuracy and area under the curve (AUC). In another study, Li, et al. (2017) applied a data-driven deep learning model for DR detection, in which fundus disease images (Lahmiri, et al., 2017) were processed and the classification model aided in separating the healthy images from the disease images.

In (Gulshan, et al., 2016), a deep learning model was developed to detect DR and macular edema from fundus images of the retina. A deep convolutional neural network (CNN) was used to generate a retinal image dataset of 128,175 images (Castellano, et al., 2020). The sensitivity and specificity metrics of this study allowed for the identification of treatable DR in diabetic patients via deep neural tissue models. Research by (Swapna, et al., 2018) used a deep learning model for classifying diabetes via heart rate variability (HRV) data. Dynamic characteristics associated with HRV data were extracted via a combination of long-term memory (LTM) and CNNs. The model results provide remarkable accuracy in detecting diabetes based on HRV data.

Most recently, Hemanth, et al. (2020) employed a hybrid technique that combines image processing and deep learning to detect and classify DR. The model was validated via the 400-image retinal fundus dataset from the MESSIDOR database with good results. Previously, Lunscher, et al. (2017) had developed a computerized sensory system to help analyse fundus images under different lighting and views. Basically, their research adopted the artificial neural network model incorporating an AdaBoost classifier for feature extraction with the data being analysed via a mixed Gaussian, SVM and kNN model to classify cases of non-retinopathic lesions.

In Reddy \& Khare $(2017 ; 2018)$, an algorithm for the classification of diabetes based on Firefly-BAT (FFBAT) optimization was developed. The unique contribution of this work is the use of the locality preserving projection (LPP) algorithm via fuzzy rules for feature extraction and the FFBAT-ANN model for classification. This combination results in better classification results, which increases the accuracy of the results. Several studies have also used probabilistic neural networks (PNNs), Bayesian classification, and SVM to classify nonproliferative (NPDR) and nonproliferative and proliferative (PDR) types of diabetic retinopathy. Haemorrhages images (Lahmiri, 2017) from a blood vessel were analysed using imaging techniques, and the features extracted from the classifier allowed the type of DR disease to be classified (Shen, et al., 2016; Kanungo, et al., 2017; Shanthi \& Sabeenian, 2019).

Altogether, the connected research indicated that much of the work on identifying DR revolves around using different machine learning (ML) models vis-à-vis their comparative effectiveness. Unfortunately, little emphasis has been given to improving the quality of the DR Dataset, which may provide more accurate results. Importantly, the reliability of the results obtained via ML models depends on the characteristics of the dataset. Extracting the most significant characteristics from the dataset and using appropriate downsizing techniques can therefore help improve the predictive accuracy of ML models. 


\section{PROPOSED METHOD}

As shown in Figure 1, the process by which an estimated diagnosis is generated is based on the analysis of different aspects of the images or retinographies. Given an image, to ensure that its high level targets do not interfere in later stages, the first step is to perform pre-processing operations, such as the enhancement of the contrast and the elimination of the area of the optical disc. Any lesion in the group of exudates is classified as a "bright lesion." Accordingly, a stage of detection of the bright areas should be applied; however, pixels with a high gloss index do not necessarily indicate an injury. To correctly determine these, a couple of stages of generation and purification of probable injuries are applied. Based on the purified maps, the classification stage is carried out by modifying the kNN grouping technique. Below, each of the characteristics of the different stages is described.

Figure 1. Flow of proposed lesion detection

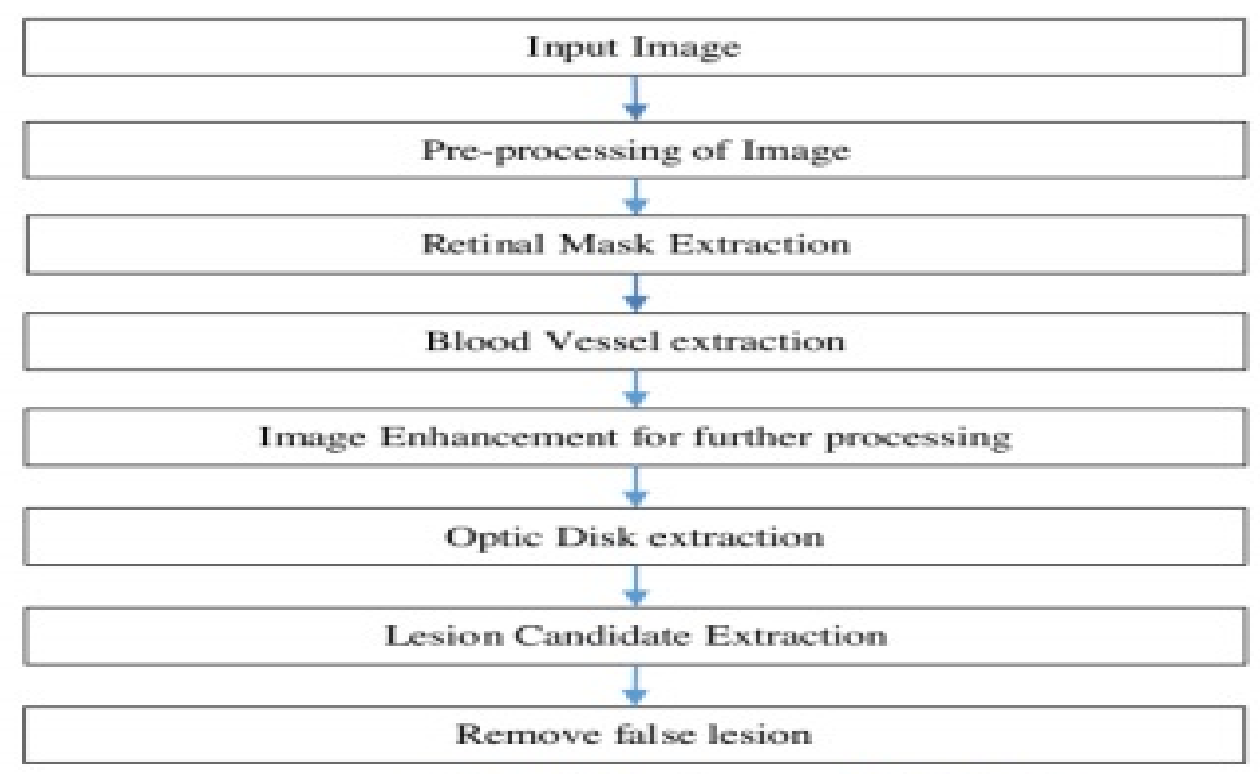

\subsection{Selection of Retinographies}

Kälviäinen (2007) provided the free fundus image repository called DIARETDB1 (Diabetic retinopathy database and evaluation protocol), which encompassed the basis for testing the method. The image bank contains 89 retinographies; 84 of these contain at least signs of non-proliferative DR and the remaining 5 are from healthy patients based on conclusions made in consensus by three specialists. These images were taken at the University Hospital of Kuopio, in Finland. Likewise, they were selected by expert physicians with their distribution corresponding to a typical population, that is, the data are partial and there is no a priori information that can be deduced from them. These images were acquired with the same field of vision, at $50^{\circ}$.

The data correspond to a practical situation, in which the images are comparable and can be used to evaluate the general performance of the proposed method. A total of 20 repository images were randomly selected. More specifically, $2 \%$ of the pixels of the said images were randomly selected and used to generate the training set of a first pixel classifier. A duplication of points was avoided, 
as well as the treatment of the pixels that were within the area of interest. The "area of interest" is understood as that area that excludes the shadow of the image and the optical disc.

A different group, constituted by 20 images randomly, was selected. These images were used to generate the training set of two additional classifiers, which differed as they assigned the values of belonging to hard exudates and to soft exudates. A total of 274 hard exudates and 131 soft exudates were part of these training sets respectively.

Algorithm 1 - Pre-processing for Contrast and Luminosity Normalization of retinal image (Foracchia, et al. 2005)

Require: The image Iwithsizeofm $\times n$.

1: Initialize the matrix $B_{n \times m}$ with zeros.

2: for $(x, y) \in I_{n \times m}$ do Determination of Background pixels

3: $N(x, y) \neg$ Neighborhood centered on $(\mathrm{x}, \mathrm{y})$

4: $\mu_{N} \leftarrow \operatorname{mean}[N(x, y)]$

5: $\sigma_{N} \leftarrow \sqrt{\operatorname{Var}[N(x, y)]}$

6. $d_{M} \leftarrow\left|\frac{I(x, y)-\mu_{N}}{\sigma_{N}}\right|$ Mahalanomis Distance

7.if $d_{M}<\tau$ then

8. $B(x, y)=1$ Pixels from the background image

9: Initialize the matrix Io $I_{n \times m}^{0}$ with zeros.

10: for $(x, y) \epsilon I_{n \times m}$ do Normalization of the pixels of the Image

11: if $B(x, y)=1$ then

12: $N_{B}(x, y) \leftarrow(x, y) \in\{N(x, y) \operatorname{suchthat} B(x, y)==1\}$

13: $L \leftarrow \operatorname{mean}\left[N_{B}(x, y)\right]$

14: $C \leftarrow \sqrt{\operatorname{Var}\left[N_{B}(x, y)\right]}$

15. $I^{0}(x, y)=\frac{I(x, y)-L}{C}$ Normalize the pixel

16: return $I^{0}$. Normalized Image

\subsection{Retinal Mask and Blood Vessel Extraction}

\section{Matched Filter}

The Matched Filter is a colour bank specially designed for the detection of blood vessels in retinal images. Take into account that the "gray" levels of the transverse section of the blood vessels are similar to the Gaussian function:

$$
K(x, y)=-\exp \frac{-x^{2}}{2 \sigma^{2}} \text { with } \frac{L}{2} \leq y \leq \frac{L}{2}
$$


Where $L$ represents the size of the vessels along the yaxis, and $\sigma$ is the deviation standard of the Gaussian.

This kernel is oriented along the y axis. For more details see Kaba, et al. (2013) and Singh \& Srivastava (2016). The detection of blood vessels will be done by rotating the blood vessels kernel in 180 directions within the range $\left[-\frac{\pi}{2}, \frac{\pi}{2}\right]$. This gives a detection probability of the blood vessels in the different orientations. In this way, the Kernel of the Matched Filter may be expressed as follows:

For the segmentation stage, two thresholds may be computed. The first one will be very restrictive (retinal mask) and will only let the pixels pass with very high intensity, which according to the result of the Matched filter, will have a very high probability of belonging to the blood vessels. The second one, being less restrictive and letting more pixels pass inside, some of which may not belong to the vessels.

These thresholds may be computed by dividing the histograms and just bypassing a certain percentage of the pixels with high intensity. For the first threshold, we will leave pass $3 \%$ of pixels with higher intensity, while for the second threshold we will let pass up to $7 \%$ of pixels with greater intensity. The results of the thresholds will be used as seed for the stage of growth of the regions. The growth of regions comprises grouping pixels according to their connectivity and if they meet certain specific property. Typically, you will start with some seed pixels and begin to add those neighbouring ones that would comply with the specific property. Therefore, using the two previous segmentations as seed and agreement if they are 8-connected, the first segmentation will grow little by little, according to the second segmentation, until forming the blood vessels.

\subsection{Optical disk Detection and Removal}

Estimation of Luminosity and Contrast drifts: Let us assume a set of background pixels $\mathfrak{B}, \hat{L}(x, y)$ and $\hat{C}(x, y)$ resulting for a single pixel:

$$
I(x, y)_{\text {enhance }} \sim \mathcal{N L}(x, y), \mathcal{C}(x, y),(x, y) \in \mathfrak{B}
$$

The estimation of these background pixels is derived by using the background component of the test image. An approximation of the test image is recovered such that:

$$
\widehat{I^{0}}(x, y)_{\text {enhance }}=\frac{I(x, y)-\hat{L}(x, y)}{\hat{C}(x, y)}
$$

While having the first possibility ( $\mathrm{C}$ and $\mathrm{L}$ are constant in $\mathrm{N}$ neighbourhood), the pixel intensity for $\mathrm{N}$ is autonomous. Accordingly, the estimation of standard deviation and mean value gives $\hat{C}(x, y)$ and $\hat{L}(x, y)$. This method needs to adapt to the similar calculation issues specified in the last paragraph. Besides, we are currently managing an inadequate set of pixels. Those which extract the use of filtering would be more problematic.

A square-preparing arrangement like the one exhibited in the past has been embraced. $\mathfrak{B}$ is the background pixels. Full $\hat{C}(x, y)$ and $\hat{L}(x, y)$ were then acquired by applying a bicubic addition on the sub-tested images. 
Multiply the Mask with Enhanced Image: After the morphological operation the mask image of the blood vessel that is achieved is represented as $I_{\text {mask }}$.

As shown in Figure 2, once masked information of blood vessel is achieved, it is multiplied with the enhanced image, yielding a blood vessel free image. Theoretically, it has been assumed that up to $25 \%$ to $30 \%$ image information is blood vessel only (e.g., Marín, et al., 2011; Patwari, et al. (2013).

Figure 2. Image Enhancement

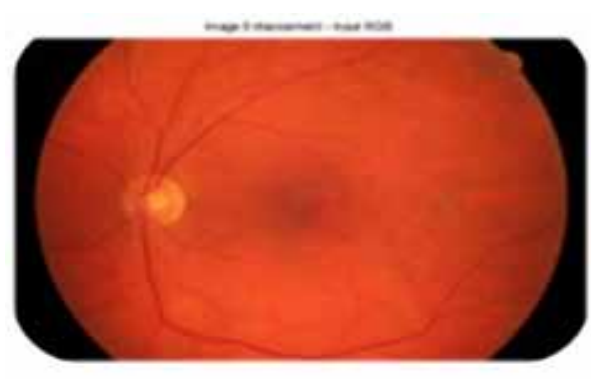

Original hpot leap

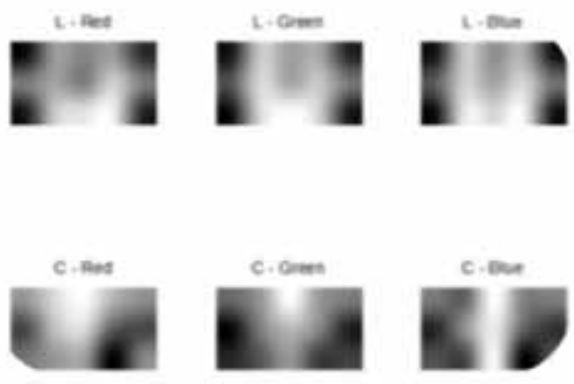

Estinated luem inosity and contrast dift

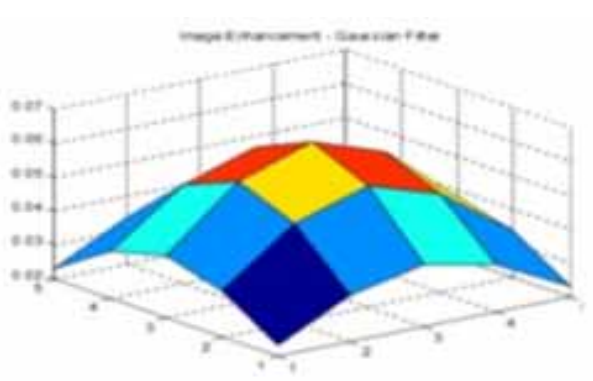

Grussian Filer

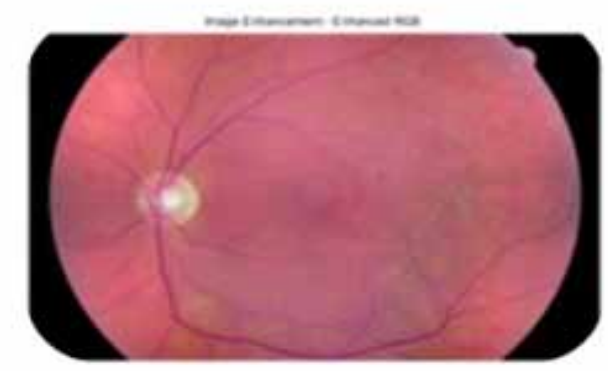

Erhare RGB
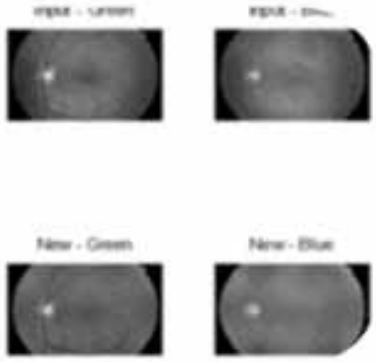

This, of course, is not desirable in optic disk detection. With the help of the above computation, it reduced the candidate region, which further needs lesser computational effort than the original one.

$$
I_{\text {candidate }}(x, y)=I_{\text {mask }}(x, y) * I_{\text {enhance }}(x, y)
$$




\subsection{Calculation of Mean, Standard Deviation and Histogram of $\mathrm{I}_{\text {candidate }}$ Subblocks}

After Estimation of luminosity and contrast drifts the $\boldsymbol{I}_{\text {candidate }}$ remains with the retina and optic disk information only. Further, a window is calculated based on database ground truth information claiming optic disk.

The size of the window may change, but it is observed that there is not much change in size according to assumption $\mathrm{W} W \cong G T{ }_{\text {optic }}$ disk. Estimated $\mathrm{W}$ is used to create the sub-block of $\boldsymbol{I}_{\text {candidate }}$. For every sub-block, there will be the calculation of mean, standard deviation and histogram. From this distribution, the mean values $\theta_{\text {mean }}$ and standard deviation $\theta_{\text {std }}$ that will be used in the following search.

$$
\theta_{\text {mean }}=\frac{1}{\mathrm{~N}} \sum_{i=1}^{N} \theta_{i}
$$

$$
\theta_{\text {std }}=\sqrt{\frac{1}{\mathrm{~N}-1}} \sum_{\mathrm{i}=1}^{\mathrm{N}}\left(\theta_{\mathrm{i}}-\theta_{\text {mean }}\right)^{2}
$$

\subsection{Optimal Threshold Calculation via Histogram}

To acquire an ideal threshold, the scanning of histogram is performed from the highest to lowest intensity-value, which is resulted from the source-image. The scanning is terminated at $T_{m}$ and $T_{s}$ intensity-levels.

Sekhar, et al. (2008) states that the position of the retinal ODE (optic disc oedema) has been endeavoured which consider the cluster of high power pixels. Sinthanayothin, et al. (1999) and Sinthanayothin, et al. (2002) suggested a technique to identify the position of the ODE (optic disc oedema) by the brightest region in image reference.

1) Segmentation Rule for Square window

$T_{m}$ : Threshold for Mean

$T_{s}:$ Threshold for std

$\mathrm{M}:$ Mean of Square window

$\mathrm{S}:$ Standard Deviation

If $\mathrm{M}<T_{m} \& \& \mathrm{~S}<T_{s}$ : Then window is Background (Black Area)

If $\mathrm{M}<T_{m} \& \& \mathrm{~S}>T_{s}$ : Then window is Retinal Background

If $\mathrm{M}>T_{m} \& \& \mathrm{~S}<T_{s}$ : Then window is Optic disk

If $\mathrm{M}>T_{m} \& \& \mathrm{~S}>T_{s}$ : Then window is Retinal Background with Exudates

2) Threshold estimation Rules

Candidate Region Detection Algorithm

Step 1: Evaluate Threshold.

Step 2: Select bright region according to threshold.

Step 3: According to area and density measures select candidate region.

Step 5: Vary threshold if candidate region is not found.

Step 6: Repeat steps 2 through 5 if threshold is greater than 0 . 
Step 7: End.

Figure 3 shows the outcome of abovementioned techniques

Figure 3. Optic Disk Region Mask

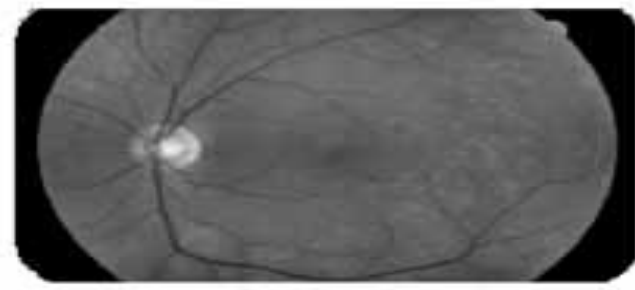

Imput green colour band

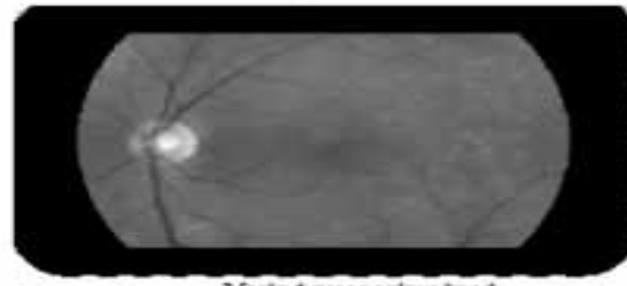

Mariad areen colour tend

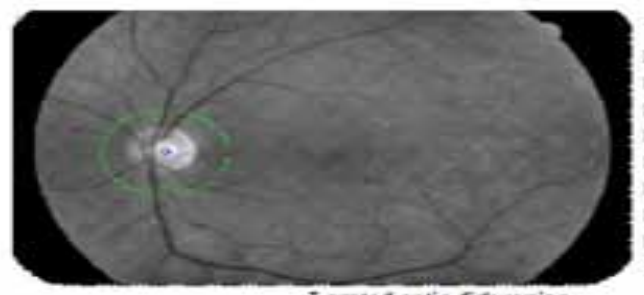

Located optic dist region

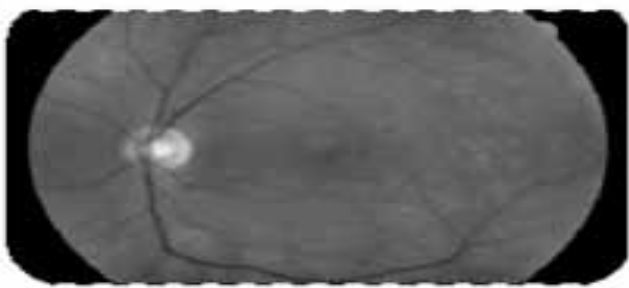

Fineted green colour band

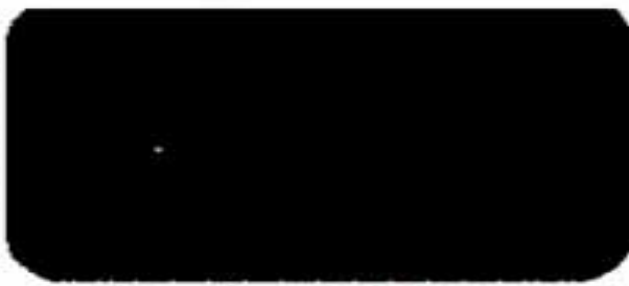

Exposent of geen colour bant

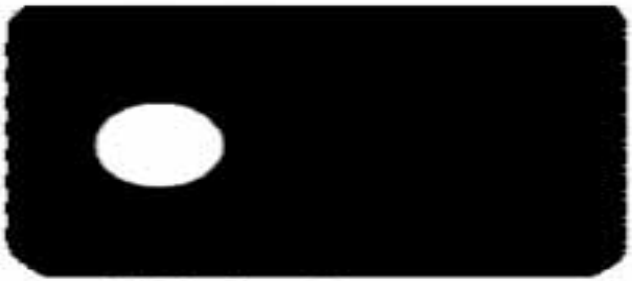

Optic dikregion mak

\subsection{Bright Lesions Detection}

One strategy is to perform a pixel-to-pixel classification on retinal images in order to detect pixels that are potentially part of a bright lesion. These regions could potentially contain exudates. The detection and classification of bright lesions, if any, is done as follows:

- Each pixel was classified, which resulted in something called "probable injury map", which indicates the probability that a pixel is part of a bright injury.

- $\quad$ Pixels with high probability were grouped in pixel cluster, probably injuries.

A k-NN algorithm with Euclidean distance and $\mathrm{k}=3$ was used. The classical philosophy of this algorithm stipulates that a new instance to be classified must be assigned the median value among the classes of the $\mathrm{k}$ instances more similar to it.

In this work, a variant of this philosophy is used to distribute the values of probability in a continuous space, assigning as value of belonging the average value between the classes of the $\mathrm{k}$ nearest instances. The new classified instances were not used as training input during the classification.

The outputs of a Gaussian filter were used as descriptors of each pixel: 
$g(x, y)=\frac{1}{\sqrt{2 \pi \sigma^{2}}} e^{-\frac{\left(x^{2}-y^{2}\right)}{2 \sigma^{2}}}$

Where $(x, y)$ are the pixel coordinates and $\sigma$ the scale. For this function we used the variants obtained from the derivatives up to the second order such that the set of filters was defined with the functions in scales $\sigma=\{1,2,3,4,8,16\}$, which generates 30 filter variants.

Figure 4 shows two of these filters.

Figure 4. (a) Gaussian, (b) Derivative with respect to $x$ of the Gaussian
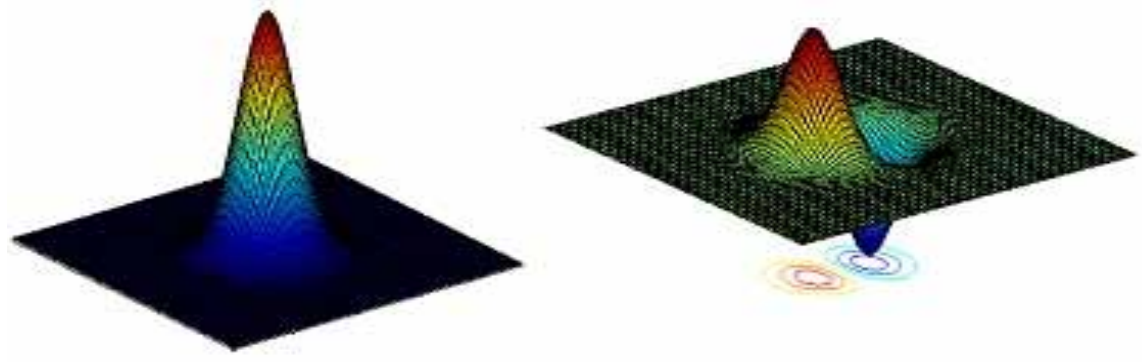

$$
\left\{g(x, y), \frac{d g(x, y)}{d x}, \frac{d g(x, y)}{d y}, \frac{d^{2} g(x, y)}{d x d y}, \frac{d^{2} g(x, y)}{d x^{2}}, \frac{d^{2} g(x, y)}{d y^{2}}\right\}
$$

Aside from aforementioned outputs of the filters, the intensity value of the pixel in the green channel was added as an extra descriptor, yielding a total of 31 characteristics to describe a pixel with which we would obtain our approximation space. This classifier was accelerated through Nvidia's CUDa technology using a 196-core GeForce GTS 450 graphics card and the Jacket framework, which implements this technology on MATLAB, resulting in an improvement of more than 100x.

On the maps of probable lesions (see Figure 5) a morphological aperture operator was applied, that is, an erosion operator followed by a morphological dilatation operator via the same structural element (a circumference of unit radius). The following equations describe the elementary operators of morphological analysis:

Dilatation:

$$
(f \oplus B)(x, y)=\max \{f(x-s, y-t) \mid(s, t) \in B\}
$$

Erosion:

$$
(f \oplus B)(x, y)=\min \{f(x+s, y+t) \mid(s, t) \in B\}
$$

The previous operation is carried out with the ultimate goal of eliminating noise resulting on the maps of probable injuries (see Figures 5 and 6). 
Figure 5. (a) and (b) Map of probable injuries (MLP)
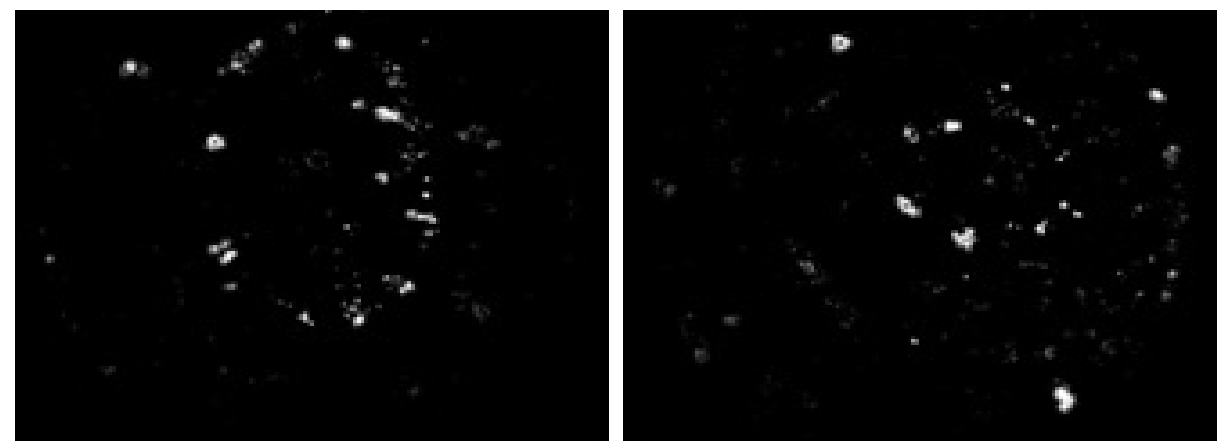

Figure 6. (a) and (b) Result after applying the opening operator and selecting the pixels with high probabilities of belonging to a bright lesion
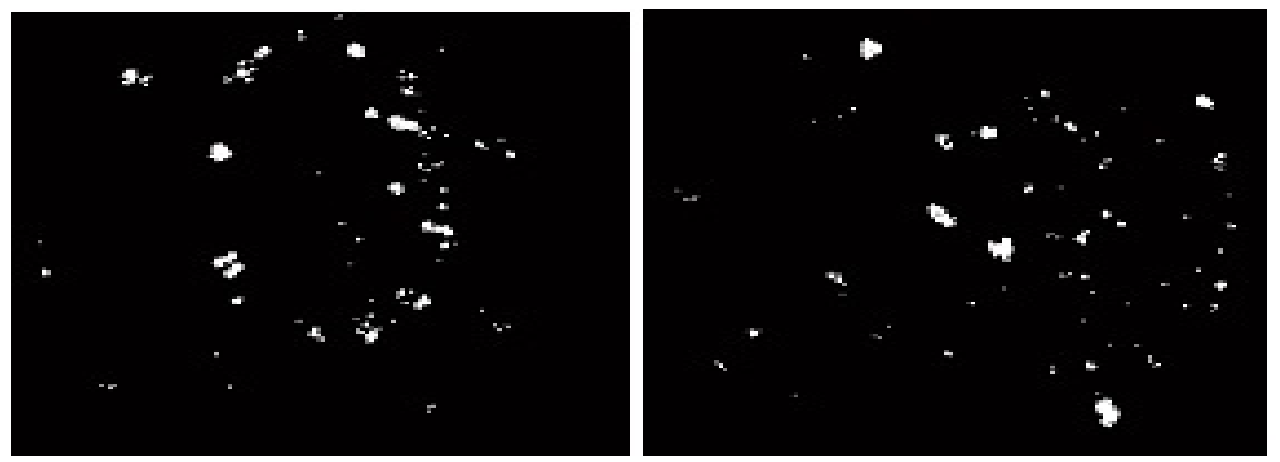

\subsection{A Classification of Bright Lesions}

A second and a third classifier $\mathrm{k}-\mathrm{NN}$ with Euclidean distance and $\mathrm{k}=1$ were used to differentiate the hard exudates and soft exudates from the bright zones. 65 lesion descriptors were used during the classification (see Table 1).

\section{SIMULATION AND RESULTS USING MATLAB 2019A}

In Figures 7(a), (b) three examples of the segmentation of the optical disk, which represent the correct location of the OD (optic disk) under different conditions of illumination and in different locations are shown. In Figure 7(a), an image covered in large part by exudates that present greater intensity than the OD is presented. Only the variance of adjacent pixels for the location of the OD. The results of the algorithm proposed in the 89 images of the database (DIARETDB1) were evaluated. While the OD was located correctly in the 89 images; yet, in 2 cases, one of them as depicted in Figure 7(b), a section of the OD was not correctly segmented. This is because the quality of the original image is very poor as represented by a large number of sub-exposed pixels in the periphery, affecting the intensity characteristic of the OD.

However, the purpose of our stage of segmentation of the optical disk is not to find exactly the edges of the OD but to extract the region approximate. With the $n$ that does not represent false positives when making the detection about the exudates, the proposed method presents a high sensitivity as it 
Table 1. The 65 descriptors used and their description

\begin{tabular}{|l|l|}
\hline \multicolumn{1}{|c|}{ Descriptor } & \multicolumn{1}{c|}{ Description } \\
\hline 1 & Area of the region \\
\hline 2 & Perimeter of the region \\
\hline 3 & eccentricity of the ellipse that best contains the region \\
\hline $4-33$ & $\begin{array}{l}\text { Average of the region through the image after applying the Gaussian filter with its respective } \\
\text { variations (see equation 8) }\end{array}$ \\
\hline $34-63$ & $\begin{array}{l}\text { Standard deviation of the regions through the image after applying the Gaussian filter with its } \\
\text { respective variations (see equation 8) }\end{array}$ \\
\hline $64-65$ & Mean and standard deviation of each of the regions in the green channel \\
\hline
\end{tabular}

Figure 7. (a) Original background image of eye and in (b) its respective pre-processed version and with the extra OD
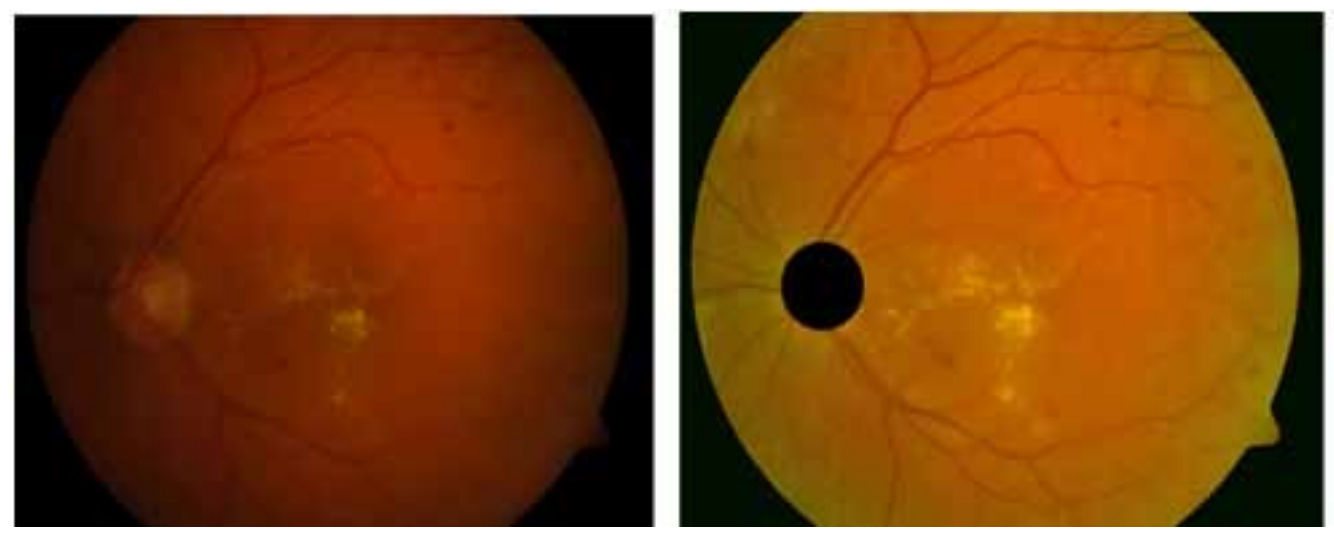

takes, in addition to the high intensity of the OD, the fact that the optical disk is crossed through the network of blood vessels. And, in their interior, there is a high density of these OD.

In testing results of the proposed algorithm in segmenting the exudates, 36 images of the DIARETDB1 database having exudates were selected. There are some images of fundus of patients previously diagnosed and treated with some type of lesion. Here, an example is shown where the patient's retina has been applied Asher therapy with the $n$ of cauterizing lesions. This type of imaging has been excluded from the analysis as the focus of this work is to contribute to the pre-diagnosis of the retinopathy to diabetes, for which the analysis of retinal images of people already diagnosed.

The evaluations made by three specialists in ophthalmology, which demarcated the regions that would potentially contain exudates, are provided in the test repository. These diagnoses were generated independently by each expert. In order to obtain a standard for comparisons, we chose to unify, in the particular case of hard exudates, the three diagnoses and then take the areas in which the three experts agreed, that is, the intercepted areas. Subsequently, we threshold these areas (see Figure 8), and call this intercept diagnosis.

In Figure 8, the acquisition of the intercept diagnosis is graphically appreciable and the sequence for obtaining it is highlighted below. For a particular type of lesion (hard or soft exudates), a threshold of the convergence areas of the diagnoses exposed by the specialists (Figure 8c) was determined with an experimentally calculated cut-off point based on a set of test images containing groups of soft and hard exudates. Results are shown in Figure 8(d). With the objective of performing an analysis of the 
Figure 8. Processing sequences up to the supposed diagnosis

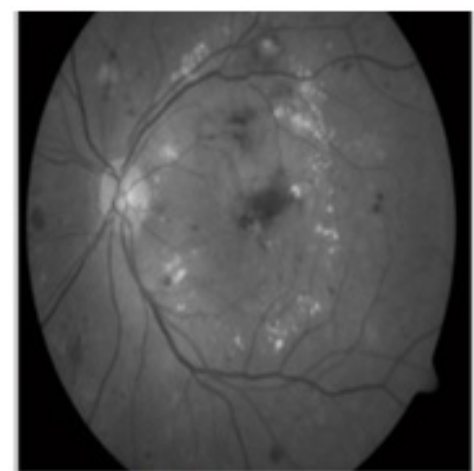

(a) Original inage

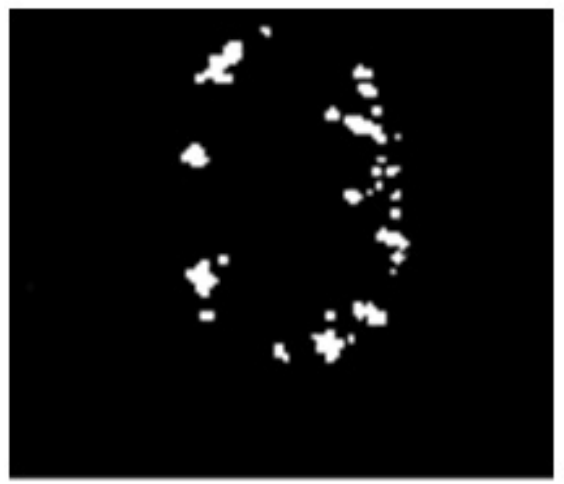

(c) Interception of the experts' diagnosis

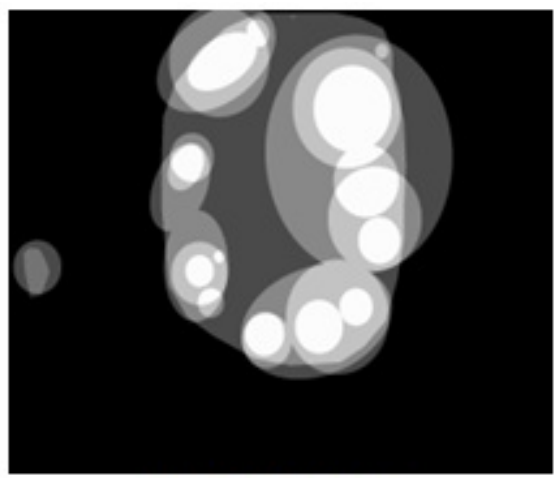

(b) Diagnosis of the expert

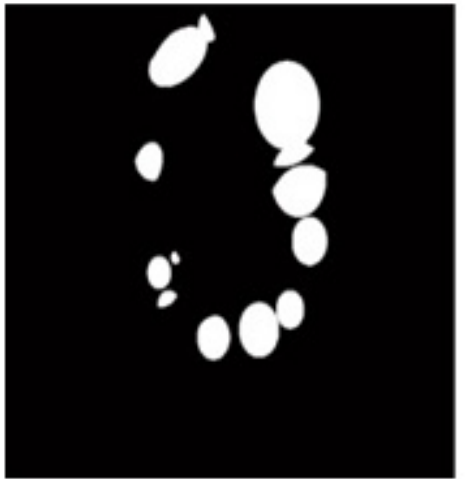

(d) Result of the thresholding and obtaining the intercept diagnosis

results in units of sensitivity (probability of labelling a pixel as part of an exudate when it really is) and specificity (probability of labelling a pixel as part of something different from an exudate when this really does not belong to an exudate), it was decided to compare against the diagnostic intercept.

In Figure 9, for each image, an intercept diagnosis was obtained and compared with the regions generated by the proposed algorithm. In the detection of hard exudates, this comparison yielded an average sensitivity of $73 \%$ and an average specificity of $99 \%$. With soft exudates, an average sensitivity of $3.4 \%$ and an average specificity of $99 \%$ was reached. Figure 10 depicts the different results obtained.

\subsection{EVALUATION FOR OPTIC DISC SEGMENTATION: DIARETDB1 AND DRIVE DATABASE}

Our optic-disc segmentation method, that is, the adaptive morphological approach, is proposed. DIARETDB1and DRIVE database are the common datasets used to test all the methods using 109 retinal images captured by fundus camera with discernable optic-disc field image reconstruction. The performance of optical disc segmentation is further analyzed with the help of O-ratio, that is, the overlapping-ratio as well as multi-modal action detection (MAD). The O-ratio is given in order to compute the common area between the optical disc region segmented by our method as well as the optic-disc region in the ground-truth. 
Figure 9. (a), c), e) Diagnostics eD g), i), k) Diagnostic eB b), d), f) Results of the detection of hard exudates, h), j), l) Results of the detection of soft exudates

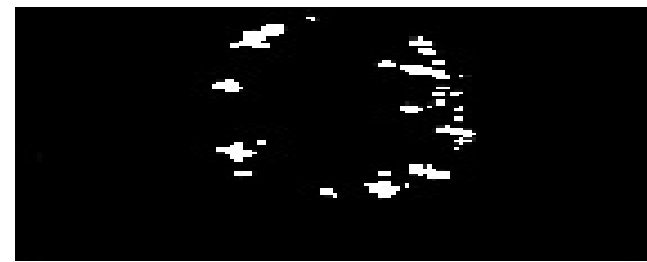

(a)

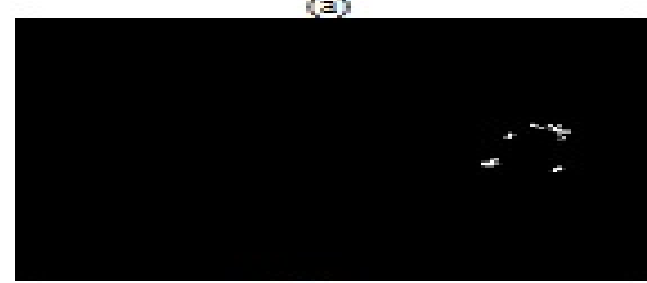

(c)

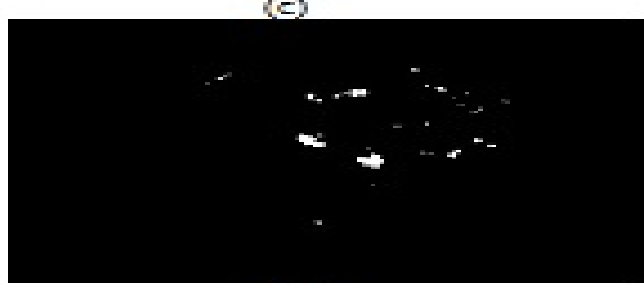

as

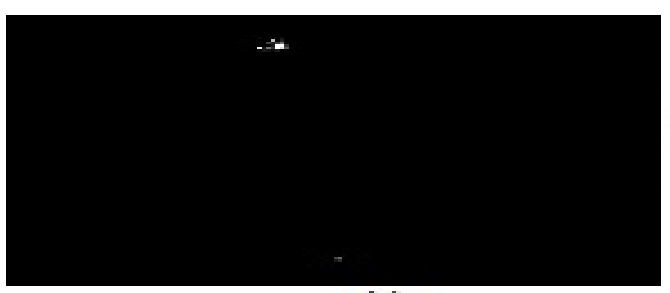

(E)

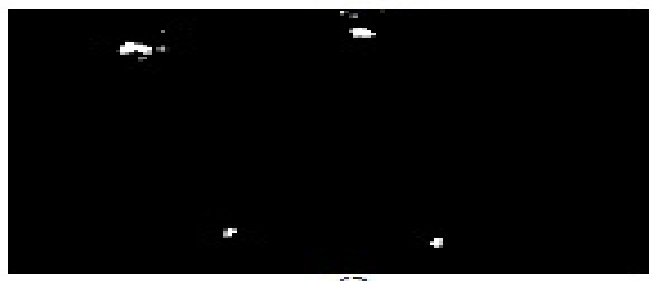

(1)

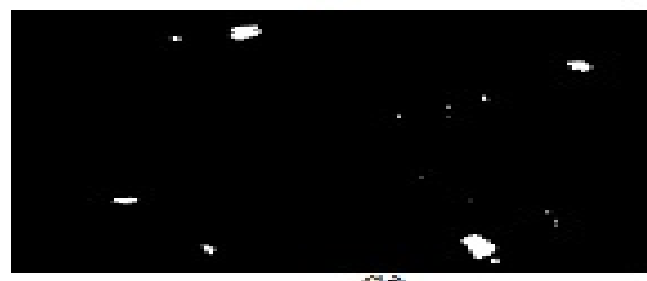

19

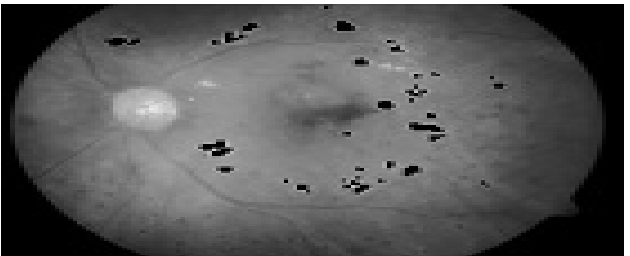

a)

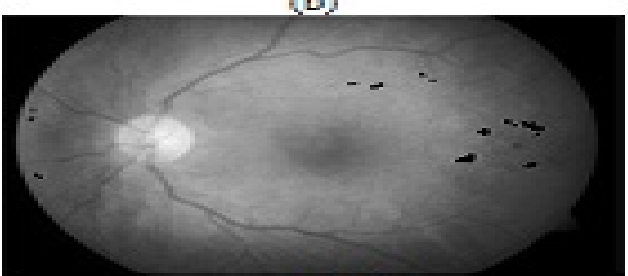

(d)

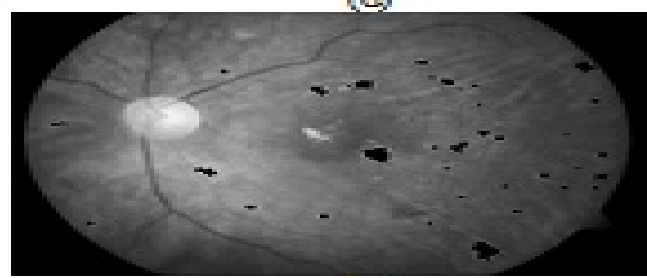

(f)

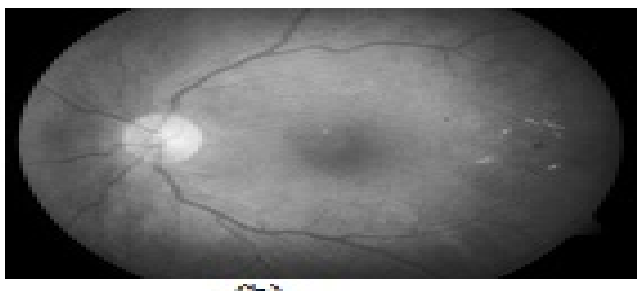

(h)

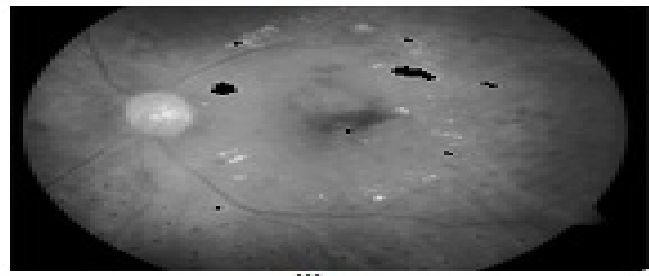

(i)

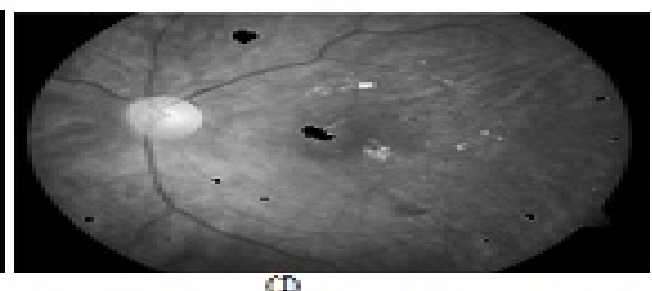


Figure 10. Various results
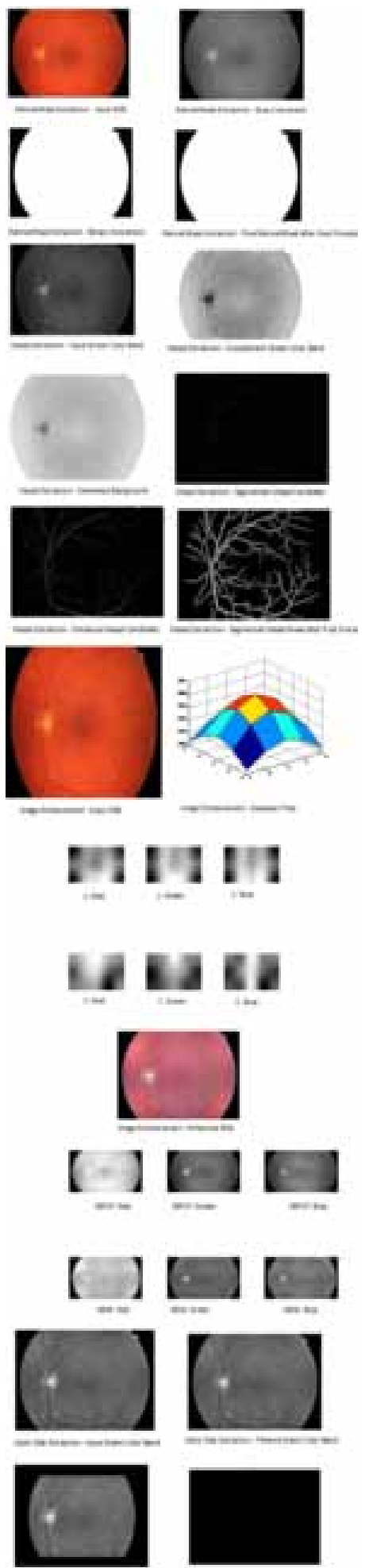
The formula of overlapping ratio is given as:

$$
\text { Oratio }=\frac{G ? S}{G ? S}
$$

Here, ' $G$ ' is the true boundary of optical disc (tagged manually) and ' $S$ ' represents the optical disc boundary achieved by proposed segmentation method. And MAD is as given by Kaba, et al. (2013):

$$
\operatorname{MAD}\left(G_{c}, S_{c}\right)=\frac{1}{2}\left\{\frac{1}{n} \stackrel{n}{i=1}_{d} d\left(g_{c i}, S\right)+\frac{1}{m} \stackrel{m}{?} d\left(s_{c i}, G\right)\right\}
$$

Also, $S_{c}$ is contour of the proposed segmented region and $G_{c}$ is the contours of ground-truth. $D\left(a_{i}, B\right)$ represents the shortest distance from the position of the pixel $a_{i}$ on the contour $A$ to $B$. A high value of O-ratio and a low value of $M A D$ is required for a good-segmentation. The sensitivity for the proposed method is tested on DIARETDB1 and DRIVE datasets as given by Kaba, et al. (2013):

$$
\text { Sensitivity }=\frac{T_{p}}{T_{p}+F_{n}}
$$

Where $T p$ and $F n$ represent true-positives and false-negatives numbers respectively. It illustrates the recognition of the foreground pixels being executed through segmentation.

Table 2 highlights the performance of the method proposed here. It also depicts the comparison results of the proposed techniques with the substitutional methods when tested on DIARETDB1 and DRIVE database images.

The Markov random fields (MRF) image-reconstruction segmentation technique reached the O-ratio (overlapping-ratio) of 0.7850 . The proposed method yielded slightly better O-ratio than MRF that is, 0.7862 and is superior to other techniques as proposed by Jung, et al. (2004). For some reasons during the performance measurement, if the MAD-value is taken into consideration, then the MRF image reconstruction method attained a lowermost value of 6.34 and hence outperformed all the other techniques. The highest average sensitivity value was 87.89 percent when tested on DIARETDB1 images.

Table 2 shows the performance of the proposed technique. It also provides the comparison results vis-à-vis other techniques. The comparison and analysis are tested on DRIVE dataset images on the basis of O-ratio, sensitivity and MAD value as compared to the Adaptive-morphologic technique (Welfer et al. 2010) as individual MAD values are not sufficient to measure the segmentationperformance.

Table 2. Performance evaluation outcome on DIARETDB1 database

\begin{tabular}{|c|c|c|c|}
\hline Method & O-R ratio (mean) & MAD (mean) & Sensitivity (mean) \\
\hline Kaba et al. (2013) & 0.7850 & 6.55 & 0.8750 \\
\hline Welfer et al. (2010) & 0.4365 & 8.31 & ---- \\
\hline Our method & 0.7862 & 6.34 & 0.8789 \\
\hline
\end{tabular}


Table 3. Performance evaluation outcome on DRIVE database

\begin{tabular}{|c|c|c|c|}
\hline Method & O-R ratio (mean) & MAD (mean) & Sensitivity (mean) \\
\hline Kaba et al. (2013) & 0.8240 & 3.39 & 0.9819 \\
\hline Welfer et al. (2010) & 0.4147 & 5.74 & ----- \\
\hline Our method & .8467 & 3.38 & 0.9834 \\
\hline
\end{tabular}

It is assumed that our algorithm with $98.34 \%$ success yields good results on DRIVE dataset as compared to DIARETDB1 dataset of $87.89 \%$.

\section{CONCLUSION}

In summary, our proposed algorithm proved to be a superior method in the detection of bright lesions, enabling the possible determination of a lesion in a colour retinography with sufficient certainty. The classification of lesions has reached satisfactory results in the differentiation of hard exudates while results obtained for the differentiation of soft exudates remain weak. Given the low level of sensitivity obtained, these results can still be improved.

Moreover, another key limitation found in this work lies in the structure of the test repository used as it does not provide precise information about the diagnosis of these lesions. As such, the method led to few diagnoses converging between the different diagnoses of the experts, with a low probability value to said injuries being assigned. Therefore, the injury classifier based on an experimental threshold may discard injuries erroneously due to low mean values.

Practical implications for the detection of optic disc and lesion location is that it will surely help the physicians to find the possibility of diabetes disease in advance.

In future work, attention is called to improve the results mainly of the soft exudate classifier and the improvement of the general performance of the algorithm. As faster retinal image acquisition often becomes necessary, the proposed algorithm can be extended and evaluated in a compressed sensing frame. Optimization of implementation time (software planning) requires special attention. 


\section{REFERENCES}

Adal, K. M., Sidibé, D., Ali, S., Chaum, E., Karnowski, T. P., \& Mériaudeau, F. (2014). Automated detection of microaneurysms using scale-adapted blob analysis and semi-supervised learning. Computer Methods and Programs in Biomedicine, 114(1), 1-10. doi:10.1016/j.cmpb.2013.12.009 PMID:24529636

Bae, J. P., Kim, K. G., Kang, H. C., Jeong, C. B., Park, K. H., \& Hwang, J. M. (2011). A study on hemorrhage detection using hybrid method in fundus images. Journal of Digital Imaging, 24(3), 394-404. doi:10.1007/ s10278-010-9274-9 PMID:20177733

Castellano, G., Castiello, C., Mencar, C., \& Vessio, G. 2020, January. Crowd detection for drone safe landing through fully-convolutional neural networks. In International Conference on Current Trends in Theory and Practice of Informatics (pp. 301-312). Springer. doi:10.1007/978-3-030-38919-2_25

Danis, R. P., \& Davis, M. D. (2008). Proliferative diabetic retinopathy. In Diabetic retinopathy (pp. 29-65). Humana Press. doi:10.1007/978-1-59745-563-3_2

Diabetic Retinopathy Study Research Group (DRSRG). (1981). Photocoagulation treatment of proliferative diabetic retinopathy: Clinical application of Diabetic Retinopathy Study (DRS) findings, DRS Report Number 8. Ophthalmology, 88(7), 583-600. doi:10.1016/S0161-6420(81)34978-1 PMID:7196564

Dougherty, E. R., \& Lotufo, R. A. (2003). Hands-on morphological image processing (Vol. 59). SPIE press. doi: $10.1117 / 3.501104$

Fleming, A. D., Philip, S., Goatman, K. A., Olson, J. A., \& Sharp, P. F. (2006). Automated microaneurysm detection using local contrast normalization and local vessel detection. IEEE Transactions on Medical Imaging, 25(9), 1223-1232. doi:10.1109/TMI.2006.879953 PMID:16967807

Foracchia, M., Grisan, E., \& Ruggeri, A. (2005). Luminosity and contrast normalization in retinal images. Medical Image Analysis, 9(3), 179-190. doi:10.1016/j.media.2004.07.001 PMID:15854840

Gulshan, V., Peng, L., Coram, M., Stumpe, M. C., Wu, D., Narayanaswamy, A., Venugopalan, S., Widner, K., Madams, T., Cuadros, J., Kim, R., Raman, R., Nelson, P. C., Mega, J. L., \& Webster, D. R. (2016). Development and validation of a deep learning algorithm for detection of diabetic retinopathy in retinal fundus photographs. Journal of the American Medical Association, 316(22), 2402-2410. doi:10.1001/jama.2016.17216 PMID:27898976

Hemanth, D. J., Deperlioglu, O., \& Kose, U. (2020). An enhanced diabetic retinopathy detection and classification approach using deep convolutional neural network. Neural Computing \& Applications, 32(3), 707-721. doi:10.1007/s00521-018-03974-0

Inbarathi, R., \& Karthikeyan, R. (2014). Detection of retinal hemorrhage in fundus images by classifying the splat features using SVM. International Journal of Innovative Research in Science, Engineering and Technology, 3(3), 1979-1985.

Jitpakdee, P., Aimmanee, P., \& Uyyanonvara, B. 2012, May. A survey on hemorrhage detection in diabetic retinopathy retinal images. In 2012 9th International Conference on Electrical Engineering/Electronics, Computer, Telecommunications and Information Technology (pp. 1-4). IEEE. doi:10.1109/ECTICon.2012.6254356

Jung, K., Kim, K. I., \& Jain, A. K. (2004). Text information extraction in images and video: A survey. Pattern Recognition, 37(5), 977-997. doi:10.1016/j.patcog.2003.10.012

Junior, S. B., \& Welfer, D. (2013). Automatic detection of microaneurysms and hemorrhages in color eye fundus images. International Journal of Computer Science and Information Technologies, 5(5), 21-37. doi:10.5121/ ijcsit.2013.5502

Kaba, A. D., Li, Y., \& Liu, X. (2013). Segmentation of blood vessels and optic disk in retinal images. IEEE J Biomed Health Inf.

Kälviäinen, R. V. J. P. H., \& Uusitalo, H. (2007). DIARETDB1 diabetic retinopathy database and evaluation protocol. Medical Image Understanding and Analysis, 61. 
Kanungo, Y. S., Srinivasan, B., \& Choudhary, S. 2017, May. Detecting diabetic retinopathy using deep learning. In 2017 2nd IEEE International Conference on Recent Trends in Electronics, Information \& Communication Technology (RTEICT) (pp. 801-804). IEEE. doi:10.1109/RTEICT.2017.8256708

Krause, J., Gulshan, V., Rahimy, E., Karth, P., Widner, K., Corrado, G. S., Peng, L., \& Webster, D. R. (2018). Grader variability and the importance of reference standards for evaluating machine learning models for diabetic retinopathy. Ophthalmology, 125(8), 1264-1272. doi:10.1016/j.ophtha.2018.01.034 PMID:29548646

Lahmiri, S. (2017). High-frequency-based features for low and high retina haemorrhage classification. Healthcare Technology Letters, 4(1), 20-24. doi:10.1049/htl.2016.0067 PMID:28529759

Lahmiri, S., \& Shmuel, A. (2017). Variational mode decomposition based approach for accurate classification of color fundus images with hemorrhages. Optics \& Laser Technology, 96, 243-248. doi:10.1016/j. optlastec.2017.05.012

Li, X., Pang, T., Xiong, B., Liu, W., Liang, P., \& Wang, T. 2017, October. Convolutional neural networks based transfer learning for diabetic retinopathy fundus image classification. In 2017 10th International Congress on Image and Signal Processing, BioMedical Engineering and Informatics (CISP-BMEI) (pp. 1-11). IEEE. doi:10.1109/CISP-BMEI.2017.8301998

Lunscher, N., Chen, M. L., Jiang, N., \& Zelek, J. (2017). Automated screening for diabetic retinopathy using compact deep networks. Journal of Computational Vision and Imaging Systems, 3(1). Advance online publication. doi:10.15353/vsnl.v3i1.182

Marín, D., Aquino, A., Gegúndez-Arias, M. E., \& Bravo, J. M. (2011). A new supervised method for blood vessel segmentation in retinal images by using gray-level and moment invariants-based features. IEEE Transactions on Medical Imaging, 30(1), 146-158. doi:10.1109/TMI.2010.2064333 PMID:20699207

Marin, D., Gegundez-Arias, M. E., Suero, A., \& Bravo, J. M. (2015). Obtaining optic disc center and pixel region by automatic thresholding methods on morphologically processed fundus images. Computer Methods and Programs in Biomedicine, 118(2), 173-185. doi:10.1016/j.cmpb.2014.11.003 PMID:25433912

Niemeijer, M., Van Ginneken, B., Cree, M. J., Mizutani, A., Quellec, G., Sánchez, C. I., Zhang, B., Hornero, R., Lamard, M., Muramatsu, C., Wu, X., Cazuguel, G., You, J., Mayo, A. I., Qin Li, , Hatanaka, Y., Cochener, B., Roux, C., Karray, F., \& Abramoff, M. D. et al. (2009). Retinopathy online challenge: Automatic detection of microaneurysms in digital color fundus photographs. IEEE Transactions on Medical Imaging, 29(1), 185-195. doi:10.1109/TMI.2009.2033909 PMID:19822469

Parvati, K., Rao, P., \& Mariya Das, M. (2008). Image segmentation using gray-scale morphology and marker-controlled watershed transformation. Discrete Dynamics in Nature and Society, 2008, 2008. doi:10.1155/2008/384346

Patwari, M. B., Manza, R. R., Rajput, Y. M., Deshpande, N. K., \& Saswade, M. (2013). Extraction of the retinal blood vessels and detection of the bifurcation points. International Journal of Computers and Applications, 77(2).

Rahim, S. S., Jayne, C., Palade, V., \& Shuttleworth, J. (2016). Automatic detection of microaneurysms in colour fundus images for diabetic retinopathy screening. Neural Computing \& Applications, 27(5), 1149-1164. doi:10.1007/s00521-015-1929-5

Rahim, S. S., Palade, V., Shuttleworth, J., \& Jayne, C. 2014, September. Automatic screening and classification of diabetic retinopathy fundus images. In International Conference on Engineering Applications of Neural Networks (pp. 113-122). Springer. doi:10.1007/978-3-319-11071-4_11

Rahim, S. S., Palade, V., Shuttleworth, J., \& Jayne, C. (2016). Automatic screening and classification of diabetic retinopathy and maculopathy using fuzzy image processing. Brain Informatics, 3(4), 249-267. doi:10.1007/ s40708-016-0045-3 PMID:27747815

Rasta, S. H., Partovi, M. E., Seyedarabi, H., \& Javadzadeh, A. (2015). A comparative study on preprocessing techniques in diabetic retinopathy retinal images: Illumination correction and contrast enhancement. Journal of Medical Signals and Sensors, 5(1), 40. doi:10.4103/2228-7477.150414 PMID:25709940

Reddy, G. T., \& Khare, N. (2017). Hybrid firefly-bat optimized fuzzy artificial neural network based classifier for diabetes diagnosis. International Journal of Intelligent Engineering and Systems, 10(4), 18-27. doi:10.22266/ ijies2017.0831.03 
Reddy, G. T., \& Khare, N. (2018). Heart disease classification system using optimised fuzzy rule based algorithm. International Journal of Biomedical Engineering and Technology, 27(3), 183-202. doi:10.1504/ IJBET.2018.094122

Sahlsten, J., Jaskari, J., Kivinen, J., Turunen, L., Jaanio, E., Hietala, K., \& Kaski, K. (2019). Deep learning fundus image analysis for diabetic retinopathy and macular edema grading. Scientific Reports, 9(1), 1-11. doi:10.1038/ s41598-019-47181-w PMID:31341220

Sekhar, S., Al-Nuaimy, W., \& Nandi, A. K. 2008, May. Automated localisation of retinal optic disk using Hough transform. In Biomedical Imaging: From Nano to Macro, 2008. ISBI 2008. 5th IEEE International Symposium on (pp. 1577-1580). IEEE. doi:10.1109/ISBI.2008.4541312

Shah, K. B., \& Han, D. P. (2004). Proliferative diabetic retinopathy. International Ophthalmology Clinics, 44(4), 69-84. doi:10.1097/00004397-200404440-00007 PMID:15577565

Shanthi, T., \& Sabeenian, R. S. (2019). Modified Alexnet architecture for classification of diabetic retinopathy images. Computers \& Electrical Engineering, 76, 56-64. doi:10.1016/j.compeleceng.2019.03.004

Shen, L., Chen, H., Yu, Z., Kang, W., Zhang, B., Li, H., Yang, B., \& Liu, D. (2016). Evolving support vector machines using fruit fly optimization for medical data classification. Knowledge-Based Systems, 96, 61-75. doi:10.1016/j.knosys.2016.01.002

Singh, N. P., \& Srivastava, R. (2016). Retinal blood vessels segmentation by using Gumbel probability distribution function based matched filter. Computer Methods and Programs in Biomedicine, 129, 40-50. doi:10.1016/j. cmpb.2016.03.001 PMID:27084319

Sinthanayothin, C., Boyce, J. F., Cook, H. L., \& Williamson, T. H. (1999). Automated localisation of the optic disc, fovea, and retinal blood vessels from digital colour fundus images. The British Journal of Ophthalmology, 83(8), 902-910. doi:10.1136/bjo.83.8.902 PMID:10413690

Sinthanayothin, C., Boyce, J. F., Williamson, T. H., Cook, H. L., Mensah, E., Lal, S., \& Usher, D. (2002). Automated detection of diabetic retinopathy on digital fundus images. Diabetic Medicine, 19(2), $105-112$. doi:10.1046/j.1464-5491.2002.00613.x PMID:11874425

Swapna, G., Vinayakumar, R., \& Soman, K. P. (2018). Diabetes detection using deep learning algorithms. ICT Express, 4(4), 243-246. doi:10.1016/j.icte.2018.10.005

Walter, T., Klein, J. C., Massin, P., \& Erginay, A. (2002). A contribution of image processing to the diagnosis of diabetic retinopathy-detection of exudates in color fundus images of the human retina. IEEE Transactions on Medical Imaging, 21(10), 1236-1243. doi:10.1109/TMI.2002.806290 PMID:12585705

Welfer, D., Scharcanski, J., Kitamura, C. M., Dal Pizzol, M. M., Ludwig, L. W., \& Marinho, D. R. (2010). Segmentation of the optic disk in color eye fundus images using an adaptive morphological approach. Computers in Biology and Medicine, 40(2), 124-137. doi:10.1016/j.compbiomed.2009.11.009 PMID:20045104

Welikala, R. A., Dehmeshki, J., Hoppe, A., Tah, V., Mann, S., Williamson, T. H., \& Barman, S. A. (2014). Automated detection of proliferative diabetic retinopathy using a modified line operator and dual classification. Computer Methods and Programs in Biomedicine, 114(3), 247-261. doi:10.1016/j.cmpb.2014.02.010 PMID:24636803

[] Yadav, S.K., Kumar, S., Kumar, B., \& Gupta, R., (2016, December). Comparative analysis of fundus image enhancement in detection of diabetic retinopathy. In 2016 IEEE Region 10 Humanitarian Technology Conference $(R 10-H T C)$ (pp. 1-5). IEEE. 
Aakanksha Mahajan is currently pursuing Ph.D in Amity University, Noida. Her area of interest includes Artificial Intelligence, Machine Learning, Healthcare. She has published 10 research papers in National and International Conferences \&Journals. She has 8 years of experience in teaching \& research.

Vasudha Vashisht, PhD., is currently working with Amity University, Uttar Pradesh as an Associate Professor and Researcher. She has completed her Ph.D. in the area of Artificial Intelligence. She has authored more than 25 Research Publications in National and International Journals and Conferences. She has filed two Patents for BCl research work and received the copyright of her research work from Govt. of India. She has also received an award of Industrial Project. She is Microsoft certified Faculty Fellow, Reviewer and Editorial Board Member of reputed International Journal(s). She has also received many Letters of Appreciation for Academic, Research and Social works. She has organized and attended many Workshops \& Training Programs, Seminars etc. She has more than 15 years of experience in University teaching \& research.

Rohit Bansal, PhD., holds a Doctorate in Management (corporate finance) from IIT Roorkee is working as an Assistant Professor in the Department of Management Studies, RGIPT, Noida, India. His teaching interest includes Financial Accounting, Security Analysis and Portfolio Management, Derivatives, Corporate Finance, Mergers \& Acquisitions, and Financial Management. He has contributed articles in peer reviewed and refereed international and Indian journals. He has reviewed core curriculum of "Finance" for Harvard Business publishing. He has contributed book chapter to book published by reputed publisher like Springer. To his credit, he has several conference paper presentations. He serves as a reviewer to many prestigious journals like, journal of Financial Risk Management published by SCRIP, China, journal of business and management, Springer, journal of economics and international finance, Wiley, Emerald, and British journal of economics, management \& trade. 